\title{
Estudo antropométrico tomográfico do quadril em uma população regional brasileira*
}

\section{Anthropometric Tomographic Study of the Hip in a Brazilian Regional Population}

\author{
Thiago Sampaio Busato ${ }^{2}$ Taiuã Verdasca Milan ${ }^{10}$ Gladyston Roberto Matioski Filho ${ }^{2(1)}$ \\ Lucas Dias Godoi2@ Marcelo Gavazzoni Morozovski2® Juan Rodolfo Vilela Capriotti2 ${ }^{20}$ \\ ${ }^{1}$ Curso de Especialização em Cirurgia do Quadril, Centro de \\ Reconstrução e Instituto de Pesquisa Articular (CRIAr), Hospital \\ Angelina Caron, Campina Grande do Sul, PR, Brasil \\ ${ }^{2}$ Grupo de quadril, Centro de Reconstrução e Instituto de Pesquisa \\ Articular (CRIAr), Hospital Angelina Caron, Campina Grande do Sul, \\ Endereço para correspondência Thiago Sampaio Busato, MD, Centro \\ de Reconstrução e Instituto de Pesquisa Articular, Hospital Angelina \\ Caron, Campina Grande do Sul, PR, Brasil \\ (e-mail: tsbusato@hotmail.com).
} PR, Brasil

Rev Bras Ortop 2022;57(2):230-240.

Trabalho desenvolvido no grupo de quadril do CRIAr - Centro de Reconstrução e Instituto de Pesquisa Articular, Hospital Angelina Caron, Campina Grande do Sul, Paraná, Brasil.
Objetivo Determinar a antropometria média do quadril de uma população regional brasileira através de medidas obtidas pela tomografia axial computadorizada (TAC). Método Análise analítico-descritiva, retrospectiva, de medidas coxofemorais de 200 TACs do abdômen de pacientes atendidos em um centro médico. Foram selecionados aleatoriamente exames que permitissem a aferição de 30 medidas antropométricas previamente definidas. Os dados foram estatisticamente analisados e comparados quanto a sexo e idade.

Resultados A prevalência de displasia do quadril foi de $6 \%$. Sinais sugestivos de impacto fêmoro-acetabular foram vistos em $26 \%$ dos casos. A análise dos resultados no grupo acima de 50 anos demonstrou medidas significativamente maiores dos: setores horizontais do acetábulo, do ângulo centro-borda e do arco acetabular, acompanhados de menor índice de extrusão, ângulo cérvico-diafisário e offset vertical. Algumas medidas foram significativamente diferentes em função do sexo: o ângulo centro-borda lateral $\left(\mu=35.5^{\circ}\right)$ e o arco acetabular $\left(\mu=68.7^{\circ}\right)$ se mostraram maiores no sexo feminino. No grupo masculino, foram maiores o índice de extrusão $(\mu=16 \%)$, o offset lateral $(\mu=38,3 \mathrm{~mm})$, a profundidade ( $\mu=19,5 \mathrm{~mm})$ e o diâmetro do colo $(\mu=26,4 \mathrm{~mm})$.

Conclusão $\mathrm{O}$ presente estudo caracterizou a antropometria do quadril de uma população brasileira. Demonstrou ainda diferenças morfológicas significativas do quadril entre diferentes faixas etárias e sexos. recebido

17 de Maio de 2020

aceito

11 de Fevereiro de 2021

Publicado on-line

Dezembro 13, 2021
DOI https://doi.org/ 10.1055/s-0041-1731658. ISSN $0102-3616$.

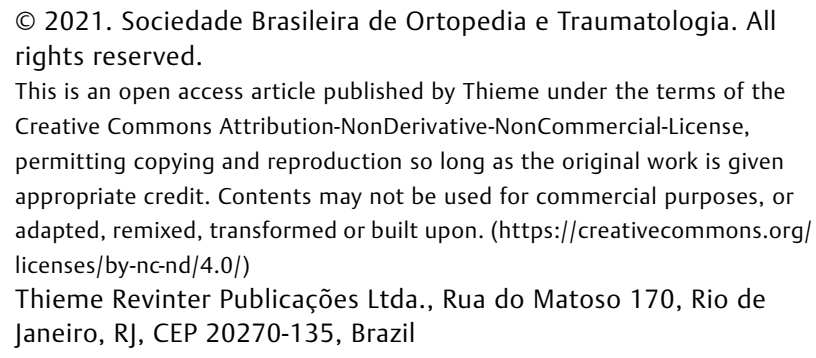

(c) 2021. Sociedade Brasileira de Ortopedia e Traumatologia. All rights reserved.

This is an open access article published by Thieme under the terms of the Creative Commons Attribution-NonDerivative-NonCommercial-License, permitting copying and reproduction so long as the original work is given appropriate credit. Contents may not be used for commercial purposes, or adapted, remixed, transformed or built upon. (https://creativecommons.org/ licenses/by-nc-nd/4.0/) Thieme Revinter Publicações Ltda., Rua do Matoso 170, Rio de Janeiro, RJ, CEP 20270-135, Brazil 
Abstract
Keywords
- anthropometry
- arthroplasty
- hip joint
- tomography, x-ray computed

Objective The present study aimed to determine the average hip anthropometry of a regional Brazilian population using measurements based on computerized axial tomography (CAT).

Methods Retrospective, descriptive analysis of hip measurements from 200 abdominal CATs from patients visiting a medical center. The tests were selected at random to determine 30 previously defined anthropometric measurements. The data were statistically analyzed and compared according to gender and age.

Results The prevalence of hip dysplasia was $6 \%$. Signs suggesting femoroacetabular impingement were seen in $26 \%$ of cases. Patients over 50 years old presented significantly greater measures of horizontal acetabulum sectors, center-edge angle, and acetabular arch, as well as lower extrusion index, cervical-diaphyseal angle and vertical offset. Some measurements were significantly different according to gender: the center to lateral edge angle $\left(\mu=35.5^{\circ}\right)$ and the acetabular arch $\left(\mu=68.7^{\circ}\right)$ were higher in females. Males presented increased extrusion index $(\mu=16 \%)$, lateral offset $(\mu=38.3 \mathrm{~mm})$, depth $(\mu=19.5 \mathrm{~mm})$, and neck diameter $(\mu=26.4 \mathrm{~mm})$.

Conclusion The present study characterized the hip anthropometry of a regional Brazilian population. It also demonstrated significant hip morphological differences per age group and gender.

\section{Introdução}

O conhecimento da antropometria do quadril, ou seja, das medidas anatômicas médias de uma determinada população é essencial. Sabe-se que as dimensões e o formato das estruturas ósseas variam, entre outros fatores, de acordo com idade, sexo e etnia. ${ }^{1}$

O domínio destas medidas permite um diagnóstico mais preciso e um melhor manejo de condições tais como o impacto fêmoro-acetabular (IFA). Outra aplicação da antropometria é no desenvolvimento de implantes para tratamento das fraturas do fêmur. ${ }^{2}$ Ressalta-se ainda, a importância deste conhecimento na artroplastia total do quadril (ATQ). Nesta, é desejável que os implantes sejam dimensionados de maneira a se acomodarem com a maior precisão possível a variabilidade anatômica dos indivíduos, haja visto que algumas das complicações resultantes da incompatibilidade das dimensões do implante com o osso receptor podem cursar até mesmo com falha precoce devido a transferência inadequada de cargas. ${ }^{2-5}$

Dada a escassez de estudos antropométricos completos sobre o quadril do brasileiro, objetivamos investigar a anatomia média da articulação do quadril de uma população regional paranaense, através de medidas coxofemorais extraídas de tomografias computadorizadas do abdômen, e compará-la com dados disponíveis na literatura sobre outras populações. Analisamos, ainda, se sexo ou idade teriam correlação significativa com a variação das medidas realizadas.

\section{Material e métodos}

Estudo retrospectivo conduzido a partir dos dados antropométricos obtidos através de 200 estudos de imagem de tomo- grafia axial computadorizada (TAC) de pacientes atendidos no centro de imagens de um hospital terciário paranaense, no período de outubro de 2014 a agosto de 2018 .

Foram incluídos, de forma aleatória, exames tomográficos de pacientes que realizaram TAC de abdômen, e que contassem com a reconstrução tomográfica dos planos axial e coronal que possibilitassem as aferições das medidas antropométricas propostas (ou seja, que contivessem cortes compreendidos entre o teto acetabular, cranialmente, até $2 \mathrm{~cm}$ abaixo da base do trocânter menor, no sentido caudal). Excluímos exames que apresentassem fraturas do quadril, presença de material de síntese ou outra situação que pudesse causar vício na coleta das medidas pretendidas, tais como tumores ósseos ou deformidades congênitas. $\mathrm{O}$ projeto de estudo foi devidamente registrado na Plataforma Brasil e aprovado pelo Comitê de Ética da instituição (CAAE 96182818.5.0000.5226).

As imagens digitalizadas foram obtidas através de tomógrafo Philips Modelo MX16EVO2 (Philips, Amsterdam, Países Baixos) 16 canais, armazenadas no sistema Picture Archiving and Communication System (PACS) Aurora (Pixeon, São Caetano do Sul, SP, Brasil). Todas as avaliações e medidas foram realizadas no programa de leitura Arya, do mesmo desenvolvedor. O quadril direito foi escolhido para todas as medições, que foram feitas pelo primeiro co-autor. Os valores encontrados foram então submetidos a tratamento estatístico e comparados com estudos semelhantes. Diagramas ilustrativos das medidas realizadas estão disponíveis nas -Figuras 1, 2 e 3.

Os parâmetros avaliados no corte coronal foram:

1. Ângulo de Sharp (índice acetabular): ângulo compreendido entre uma linha horizontal padrão (bi-isquiática) e 


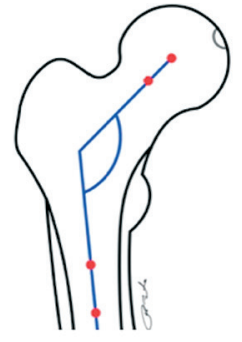

Ângulo cérvicodiafisário

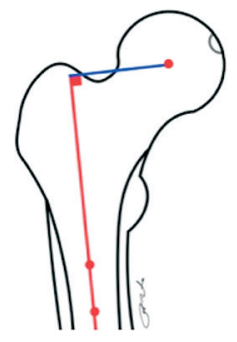

Offset lateral

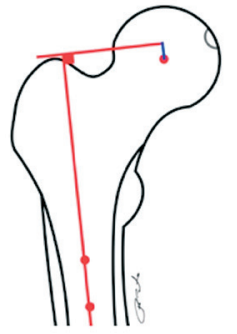

Offset Vertical

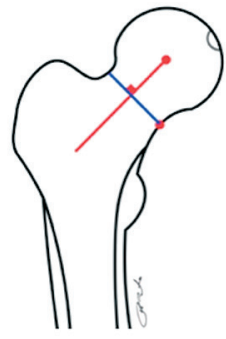

Diâmetro do colo

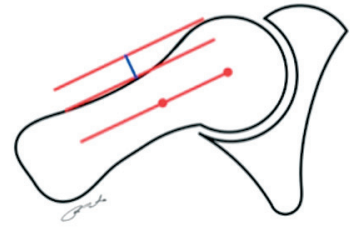

Offset cabeça-colo

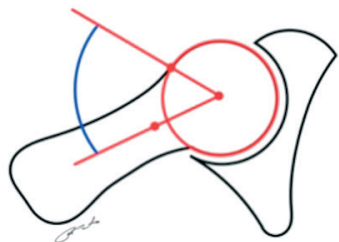

Ângulo alfa anterior

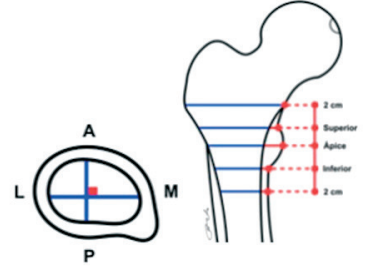

Diâmetros AP e ML do canal (em 5 cortes)

Fig. 1 Ilustração das medidas femorais.

outra linha que conecta as extremidades acetabulares ínfero-medial a súpero-lateral em seu maior diâmetro. ${ }^{6-8}$

2. Ângulo de Tönnis: ângulo compreendido entre uma linha horizontal padrão e outra linha traçada do ponto mais medial ao ponto mais lateral da sobrancelha. ${ }^{1,8-10}$

3. Profundidade acetabular: distância ortogonal do ponto médio da maior linha que conecta as extremidades acetabulares ínfero-medial e súpero-lateral para o fundo do acetábulo, em seu maior diâmetro. ${ }^{8,11}$

4. Ângulo de Wiberg (centro borda lateral): ângulo entre uma linha traçada verticalmente através do centro da cabeça femoral e outra linha traçada a partir do centro da cabeça do fêmur para a borda mais lateral da sobrancelha. ${ }^{12,13}$

5. Centro borda medial: ângulo entre uma linha vertical traçada através do centro da cabeça do fêmur e outra linha que liga o centro da cabeça do fêmur a borda mais medial da sobrancelha. ${ }^{9,14,15}$

6. Arco acetabular: Somatória dos ângulos centro borda lateral e centro borda medial. ${ }^{16,17}$

7. Ângulo Delta (Notzli): ângulo entre uma linha traçada do centro da cabeça do fêmur até a parte mais medial da sobrancelha e outra linha traçada do centro da cabeça até a parte mais lateral da fóvea da cabeça do fêmur, no

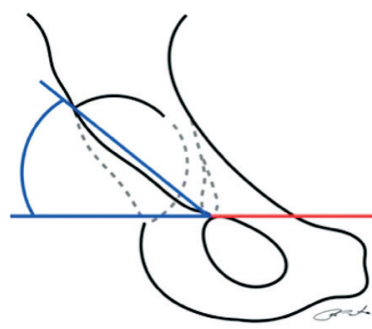

Sharp

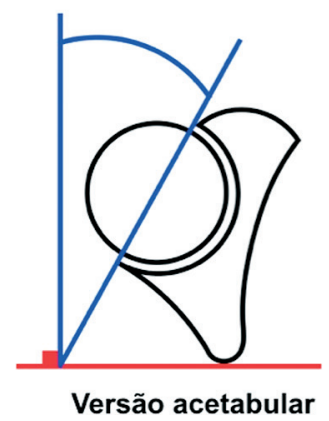

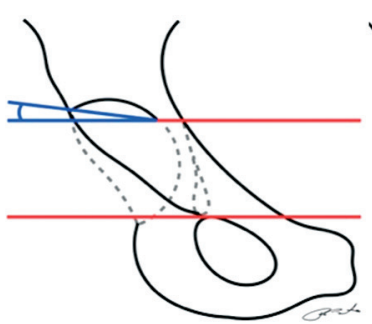

Tönnis

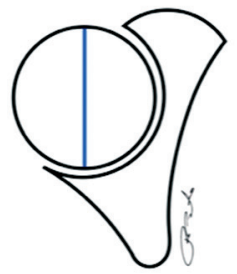

Diâmetro cefálico

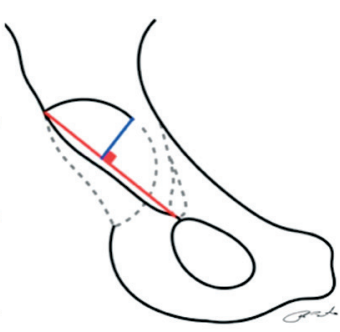

Profundidade

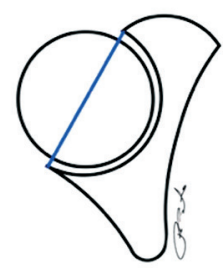

Diâmetro acetabular

Fig. 2 Ilustração das medidas acetabulares. 


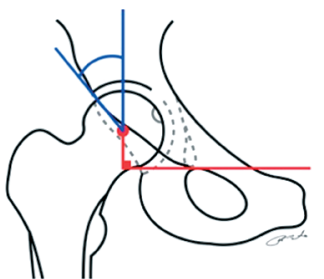

Ângulo centro borda lateral

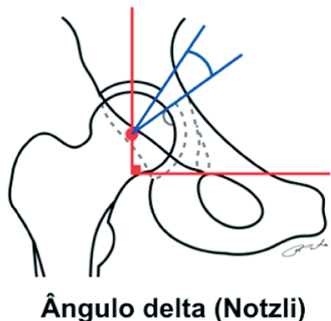

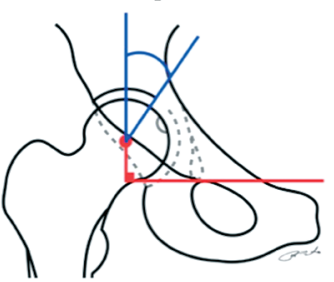

Ângulo centro borda medial

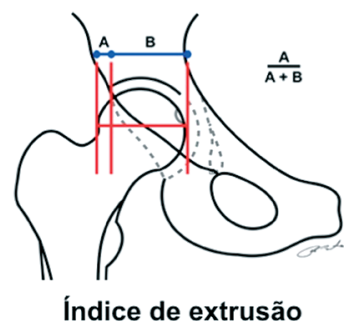

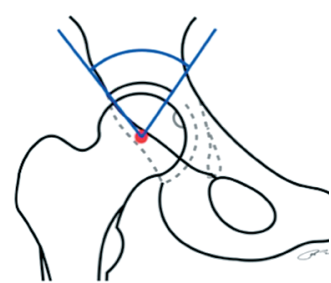

Arco acetabular

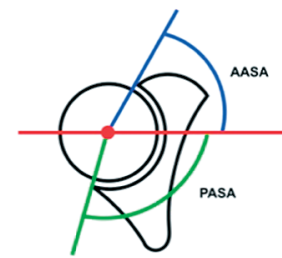

AASA+PASA $=$ HASA

Fig. 3 Ilustração das medidas combinadas.

corte tomográfico aonde esta encontra-se mais profunda. $^{18}$

8. Índice de extrusão da cabeça: medido no maior diâmetro da cabeça do fêmur. É determinado através de linhas verticais ortogonais a uma linha horizontal padrão, traçadas através da borda mais lateral da sobrancelha e da borda mais lateral da cabeça do fêmur. O índice é obtido dividindo-se a medida horizontal da parte extrusa pelo diâmetro da cabeça. ${ }^{16}$

9. Ângulo cérvico-diafisário: ângulo entre o eixo anatômico do fêmur (traçado através de pontos no centro do diâmetro médio-lateral da diáfise em duas regiões distintas) e o eixo do colo (traçado entre um ponto central do diâmetro crânio-caudal do colo do fêmur e o centro de rotação da cabeça). ${ }^{1,2,7}$

10. Offset lateral: distância ortogonal do centro de rotação da cabeça do fêmur ao eixo anatômico do mesmo osso. $^{11,12}$

11. Offset vertical: distância ortogonal do centro da cabeça femoral até uma linha que tangencia a extremidade mais cranial do trocânter maior. ${ }^{12}$

12. Diâmetro do colo do fêmur: diâmetro endosteal ortogonal ao eixo do colo, medido no corte de maior diâmetro deste. $^{10}$

13. Diâmetro do canal femoral: diâmetro endosteal médiolateral do canal do fêmur. Medido em cinco pontos, a saber: $2 \mathrm{~cm}$ acima da borda cranial do trocânter menor, na altura da borda cranial do trocânter menor, na altura do ápice do trocânter menor, na altura da borda caudal do trocânter menor e $2 \mathrm{~cm}$ distais a borda caudal do trocânter menor. ${ }^{2}$

Os parâmetros avaliados no corte axial foram:

1. Versão acetabular: ângulo entre as extremidades das paredes acetabulares anterior e posterior e uma linha ortogonal a outra linha padrão que conecta as margens pélvicas posteriores, ao nível do maior diâmetro da cabeça. ${ }^{13,14}$

2. Ângulo do setor anterior do acetábulo (AASA): ângulo entre a linha que passa nos centros das cabeças (em seu maior diâmetro) e outra linha traçada do centro da cabeça a extremidade da parede anterior. ${ }^{15}$

3. Ângulo do setor posterior do acetábulo (PASA): ângulo entre a linha que passa nos centros das cabeças (em seu maior diâmetro) e outra linha traçada do centro da cabeça a extremidade da parede posterior. ${ }^{15}$

4. Ângulo do setor horizontal do acetábulo (HASA): Somatória: AASA + PASA. ${ }^{15}$

5. Diâmetro da cabeça do fêmur: no maior diâmetro da cabeça do fêmur. ${ }^{10}$

6. Diâmetro do acetábulo: no corte de maior diâmetro da cabeça. ${ }^{10}$

7. Offset cabeça-colo ${ }^{10}$ : são traçadas três linhas paralelas: 1) eixo do colo do fêmur. 2) linha paralela à primeira, que tangencia a cortical anterior do colo. 3 ) linha paralela às demais, que tangencia a cortical anterior da cabeça do fêmur. 0 offset é dado pela distância entre as linhas 2 e 3.

8. Ânguloalfa anterior: ângulo entre o eixo do colo do fêmur até uma linha que conecta o centro de rotação da cabeça do fêmur ao seu ponto de perda da esfericidade. ${ }^{16,19}$

9. Diâmetro do canal femoral: diâmetro ântero-posterior do canal do fêmur. Medido em cinco pontos: $02 \mathrm{~cm}$ acima do trocânter menor, na altura da borda superior do trocânter menor, no ápice do trocânter menor, naborda inferior do trocânter menor e $02 \mathrm{~cm}$ distais ao trocânter menor. ${ }^{2}$

Os dados coletados foram submetidos ao teste de Kolmogorov-Smirnov para verificar a normalidade da distribuição dos valores antropométricos. $\mathrm{O}$ teste de Bartlett foi utilizado para testar se as variâncias de $K$ nos grupos (tipos de medidas antropométricas) eram homogêneas. O teste Qui-quadrado para variáveis categóricas foi realizado com a finalidade de identificar as tendências quanto a idade e sexo e o teste 
234 Estudo antropométrico tomográfico do quadril em uma população regional brasileira Busato et al.

análise de variância (ANOVA) foi utilizado para comparação de médias (considerando $p<0,05$ como significativo).

\section{Resultados}

O presente estudo analisou dados de TAC de 200 pacientes. Em cada caso foram coletadas 30 medidas, totalizando 6.000 dados para a análise estatística.

A - Tabela 1 contém os dados demográficos da população estudada. Os pacientes possuem idade média igual a 49 anos $(\mu=48,90)$, variando com desvio padrão de \pm 20 anos $(\sigma=$ $\pm 20.25)$. A composição étnica média, autodeclarada, da população que faz uso desta unidade hospitalar foi retirada do sistema de internamento, e é composta da seguinte maneira:
Tabela 1 Caracterização demográfica da amostra

\begin{tabular}{|l|l|l|l|}
\hline Caracterização & $\mathbf{n}$ & $\%$ \\
\hline \multirow{3}{*}{ Faixa Etária } & $\geq 50$ & 96 & $48 \%$ \\
\cline { 2 - 4 } & $<50$ & 104 & $52 \%$ \\
\hline \multirow{2}{*}{ Sexo } & $\mathrm{F}$ & 86 & $43 \%$ \\
\cline { 2 - 4 } & $\mathrm{M}$ & 114 & $57 \%$ \\
\hline
\end{tabular}

Fonte: Protocolo de pesquisa (2019).

$69,2 \%$ se identificam como brancos, $4,4 \%$ como negros e $26,4 \%$ como pardos.

A - Tabela 2 descreve os achados médios, mínimo e máximo da amostra geral, bem como seus desvios padrões.

Tabela 2 Medidas antropométricas da amostra geral

\begin{tabular}{|c|c|c|c|c|}
\hline Medida radiológica & Média & Desvio padrão & Mínimo & Máximo \\
\hline Sharp (ângulo) & 40,3 & 4,8 & 27,0 & 63,0 \\
\hline Tönnis (ângulo) & 3,4 & 6,8 & $-13,0$ & 50,0 \\
\hline Profundidade (mm) & 19,0 & 2,9 & 11,0 & 30,0 \\
\hline CE lateral (ângulo) & 33,8 & 8,3 & 15,0 & 64,0 \\
\hline CE medial (ângulo) & 33,4 & 8,6 & 13,0 & 66,0 \\
\hline Arco acetabular (ângulo) & 66,9 & 11,2 & 38,0 & 103,0 \\
\hline Âng. delta (ângulo) & 27,8 & 8,8 & 5,0 & 52,0 \\
\hline Índ. extrusão (\%) & 14,0 & 9,8 & 0,0 & 52,0 \\
\hline Âng. cérv. diafisário & 129,4 & 5,8 & 116,0 & 145,0 \\
\hline Offset lateral (mm) & 37,6 & 3,4 & 28,0 & 45,0 \\
\hline Offset vertical (mm) & $-1,3$ & 8,8 & $-16,0$ & 27,0 \\
\hline Diâmetro do colo (mm) & 25,2 & 4,0 & 17,0 & 37,0 \\
\hline Versão acetabular (ângulo) & 21,2 & 6,5 & 7,0 & 47,0 \\
\hline AASA (ângulo) & 63,3 & 9,7 & 26,0 & 92,0 \\
\hline PASA (ângulo) & 105,9 & 14,0 & 71,0 & 159,0 \\
\hline HASA (ângulo) & 169,4 & 19,5 & 129,0 & 252,0 \\
\hline Diâmetro cefálico (mm) & 41,8 & 4,0 & 32,0 & 52,0 \\
\hline Diâmetro acetabular (mm) & 52,4 & 4,0 & 41,0 & 61,0 \\
\hline Offset cabeça-colo (mm) & 9,4 & 2,7 & 4,0 & 28,0 \\
\hline Ângulo alfa anterior (ângulo) & 47,9 & 7,2 & 30,0 & 71,0 \\
\hline AP: $+2 \mathrm{~cm}(\mathrm{~mm})$ & 34,9 & 6,0 & 20,0 & 55,0 \\
\hline $\mathrm{ML}:+2 \mathrm{~cm}(\mathrm{~mm})$ & 40,6 & 6,3 & 22,0 & 62,0 \\
\hline AP: superior (mm) & 31,0 & 4,8 & 20,0 & 45,0 \\
\hline ML: superior (mm) & 36,8 & 4,4 & 24,0 & 50,0 \\
\hline AP: ápice (mm) & 27,4 & 5,0 & 17,0 & 40,0 \\
\hline ML: ápice (mm) & 37,6 & 5,9 & 16,0 & 51,0 \\
\hline AP: inferior (mm) & 22,6 & 4,0 & 12,0 & 35,0 \\
\hline ML: inferior (mm) & 24,4 & 5,0 & 15,0 & 42,0 \\
\hline AP: $-2 \mathrm{~cm}(\mathrm{~mm})$ & 17,8 & 3,3 & 10,0 & 28,0 \\
\hline $\mathrm{ML}:-2 \mathrm{~cm}(\mathrm{~mm})$ & 18,0 & 3,4 & 11,0 & 38,0 \\
\hline
\end{tabular}

Abreviaturas: AASA, ângulo do setor anterior do acetábulo; AP: Medida ânteroposterior; ML: Medida médio-lateral; HASA, ângulo do setor horizontal do acetábulo; PASA, ângulo do setor posterior do acetábulo.

Fonte: Protocolo de pesquisa (2019). 
De modo geral, as medidas possuem valores médios considerados normais.

Considerando o ângulo centro-borda lateral ou anterior menor do que $20^{\circ}$ como sugestivo de DDQ tivemos 12 quadris na amostra, com uma prevalência de $6 \%$. Se considerarmos a elevação do ângulo alfa acima de $55^{\circ}$ como suspeita de IFA do tipo cam, tivemos 22 quadris, ou $11 \%$. Se considerarmos o ângulo de Tönnis negativo como sugestivo de IFA do tipo pincer, tivemos 23 casos ou 11,5\%. Casos de alteração sugestiva do tipo misto responderiam por 3,5\% desta amostra. Desta maneira, $26 \%$ da amostra possui algum sinal sugestivo de IFA.

A - Tabela 3 demonstra as medições por faixa etária $(<50$ anos ou $\geq 50$ anos). Em negrito estão destacadas as medidas com diferenças estatisticamente significativas $(p<0,05)$. Algumas medidas antropométricas se mostraram significativamente diferentes em função dos grupos etários.

Nos pacientes acima de 50 anos, o ângulo de Sharp se mostrou maior, com média igual a $41,5^{\circ}$; assim como a média do CE lateral $(\mu=35,9 \mathrm{~mm})$. Da mesma maneira, a medida do arco acetabular foi maior $\left(\mu=68,6^{\circ}\right)$. Nas medidas do corte axial, observou-se também que as médias da versão acetabular $\left(\mu=22.6^{\circ}\right)$, dos ângulos AASA $\left(\mu=65.4^{\circ}\right)$, PASA $\left(\mu=110.4^{\circ}\right)$ e HASA $\left(\mu=176^{\circ}\right)$ se mostraram significativamente mais elevadas no grupo de pacientes de maior idade.

Nos mais jovens, verifica-se que o índice de extrusão apresentou média mais elevada $(\mu=16,7 \%$ ), assim como o ângulo cérvico diafisário que também se mostrou mais elevado neste grupo $\left(\mu=130,2^{\circ}\right)$.

\section{Resultados por sexo}

A - Tabela 4 demonstra as medidas obtidas por sexo. Em negrito estão destacadas as medidas com diferenças estatisticamente significativas $(p<0,05)$. Observamos que algumas medidas se mostraram significativamente diferentes.

No sexo feminino, observa-se que o ângulo médio da versão acetabular se mostrou significativamente mais elevado $\left(\mu=22.5^{\circ}\right)$ assim como o ângulo centro-borda lateral $\left(\mu=35.5^{\circ}\right)$ e $o$ arco acetabular $\left(\mu=68.7^{\circ}\right)$.

No grupo masculino, foram mais elevados o índice de extrusão $(\mu=16 \%)$, o offset lateral $(\mu=38,3 \mathrm{~mm})$, a profundidade média $(\mu=19,5 \mathrm{~mm})$ e o diâmetro médio do colo $(\mu=26,4 \mathrm{~mm})$. Ainda, os valores do diâmetro médio da cabeça $(\mu=43,6 \mathrm{~mm})$ e do diâmetro médio do acetábulo $(\mu=54,2 \mathrm{~mm})$ também se mostraram mais elevados.

$\mathrm{Na}$ - Tabela 5 vemos as medidas relacionadas ao diâmetro do canal. Observa-se que o grupo masculino apresentou medidas significativamente mais elevadas do que o grupo feminino. A - Tabela 6 demonstra que não houve diferença significativa nas medidas do canal femoral comparando-se os grupos em relação a idade.

\section{Discussão}

Neste estudo, analisamos exames de TAC de abdômen, que continham segmentações ao nível da articulação coxofemoral, para que pudéssemos caracterizar as medidas antropométricas médias desta amostra. Foram usados estu- dos de TAC do abdômen de modo a diminuir o viés de seleção, já que provavelmente não foram feitos por queixas ortopédicas (e não avaliamos sintomatologia prévia neste estudo).

O conceito moderno de impacto fêmoro-acetabular (IFA) foi descrito por Ganz e sub-dividido em três tipos: pincer, quando ocorre devido a alterações acetabulares tais como coxa profunda ou retroversão global ou focal; came, quando as alterações são femorais, geralmente devido a perda do offset cabeça-colo; e misto, o mais comum.

Ao investigar a prevalência de anormalidades ósseas predisponentes ao IFA em indivíduos assintomáticos, Kang et al. ${ }^{6}$ observaram que pacientes que apresentavam ângulo de versão acetabular variando entre 5 a $29^{\circ}$, com uma média de $18^{\circ}$, valor este que se encontra abaixo do encontrado em nossa amostra, que variou entre 7 a $47^{\circ}$, resultando em uma média de $21,2^{\circ}$. Outros autores avaliaram medidas que poderiam sugerir IFA. Uma medida amplamente descrita na literatura é o ângulo centro-borda de Wiberg. Neste mesmo estudo, ${ }^{6}$ foram encontrados valores que variaram entre 21 a $46^{\circ}$, com média de $34^{\circ}$. Em uma série de casos avaliados por Murtha et $\mathrm{al}^{8}{ }^{8}$, este ângulo variou entre $8,5 \mathrm{e}$ $32,3^{\circ}$. Em nossa amostra, encontramos medidas que variaram de 15 a $64^{\circ}$, com média de $33,8^{\circ}$.

Tannast et al. ${ }^{16}$ classificaram os acetábulos conforme esta medida em 4 grupos, nos quais ângulos $<22^{\circ}$ corresponderiam a quadris displásicos, 23 a 33 quadris normais, 34 a 39 quadris com sobrecobertura e $>40^{\circ}$ sobrecobertura severa. Na amostra estudada, a prevalência de displasia do desenvolvimento do quadril foi de $6 \%$, enquanto $26 \%$ dos casos apresentavam algum sinal sugestivo de IFA. Sobre a alta prevalência de sinais sugestivos de IFA na amostra estudada, é de extrema importância ressaltar que o diagnóstico da síndrome do IFA não depende apenas de achados de imagem e, como não coletamos dados acerca da presença de sintomatologia nestes pacientes, este achado deve ser apenas considerado do ponto de vista morfológico, e não patológico.

Quando analisados por sexo, Lepaage-Saucier et al. ${ }^{14} \mathrm{e}$ Kang et al. ${ }^{6}$ encontraram maiores médias no ângulo centroborda em homens do que em mulheres; já apenas o estudo de Mineta et al., ${ }^{10}$ curiosamente, não encontrou diferenças entre os gêneros. Nossa amostra foi condizente com a literatura prévia e evidenciou ângulos maiores em pacientes do sexo feminino quando comparados aos do sexo masculino, sendo estes $35,5^{\circ}$ e $32,6^{\circ}$, respectivamente. Kang et al. também descreveram medidas do ângulo alfa, com média de $45,5^{\circ}$, semelhantes ao nosso estudo, com média de $47,9^{\circ}$.

Sobre o ângulo delta, descrito como um sinal de displasia, Beltran et al relataram valores médios de $22,7^{\circ}$ com DP de $12,6^{\circ}$ em quadris normais. Em nosso trabalho, a média foi de $27,8^{\circ}$ com desvio padrão de $8,8^{\circ}$, resultado provavelmente relacionado com a maior cobertura acetabular em relação a outros estudos previamente citados.

Em relação ao ângulo de Tönnis, Lepaage-Saucier et al. ${ }^{14}$ relataram uma angulação média de $6^{\circ}$ sem diferenças entre os sexos; já Mineta et al..$^{10}$ encontraram médias mais baixas entre em homens e pacientes idosos. No presente estudo não encontramos diferenças significativas entre as idades. 
236 Estudo antropométrico tomográfico do quadril em uma população regional brasileira Busato et al.

Tabela 3 medidas antropométricas em função da faixa etária

\begin{tabular}{|c|c|c|c|c|c|c|}
\hline Variável & Idade & Média & Desvio padrão & Mínimo & Máximo & Valor de $p$ \\
\hline \multicolumn{7}{|l|}{ Corte coronal } \\
\hline \multirow[t]{2}{*}{ SHARP } & $\geq 50$ & 39,1 & 5,5 & 27,0 & 63,0 & \multirow[t]{2}{*}{$0.000^{*}$} \\
\hline & $<50$ & 41,5 & 3,8 & 28,0 & 49,0 & \\
\hline \multirow[t]{2}{*}{ TONNIS } & $\geq 50$ & 3,4 & 6,8 & $-12,0$ & 50,0 & \multirow[t]{2}{*}{0.977} \\
\hline & $<50$ & 3,4 & 6,8 & $-13,0$ & 35,0 & \\
\hline \multirow[t]{2}{*}{ PROFUNDIDADE } & $\geq 50$ & 18,7 & 2,8 & 12,0 & 28,0 & \multirow[t]{2}{*}{0.187} \\
\hline & $<50$ & 19,3 & 3,1 & 11,0 & 30,0 & \\
\hline \multirow[t]{2}{*}{ CENTRO-BORDA LATERAL } & $\geq 50$ & 35,9 & 8,1 & 22,0 & 62,0 & \multirow[t]{2}{*}{$0.001^{*}$} \\
\hline & $<50$ & 31,9 & 8,0 & 15,0 & 64,0 & \\
\hline \multirow[t]{2}{*}{ CENTRO-BORDA MEDIAL } & $\geq 50$ & 32,6 & 7,1 & 13,0 & 50,0 & \multirow[t]{2}{*}{0.188} \\
\hline & $<50$ & 34,2 & 9,7 & 19,0 & 66,0 & \\
\hline \multirow[t]{2}{*}{ ARCO ACETABULAR } & $\geq 50$ & 68,6 & 10,7 & 47,0 & 103,0 & \multirow[t]{2}{*}{$0.041^{*}$} \\
\hline & $<50$ & 65,4 & 11,4 & 38,0 & 103,0 & \\
\hline \multirow[t]{2}{*}{ ÂNGULO DELTA } & $\geq 50$ & 28,3 & 8,8 & 5,0 & 52,0 & \multirow[t]{2}{*}{0.409} \\
\hline & $<50$ & 27,3 & 8,8 & 5,0 & 42,0 & \\
\hline \multirow[t]{2}{*}{ ÍNDICE DE EXTRUSÃO } & $\geq 50$ & 11,2 & 8,6 & 0,0 & 34,0 & \multirow[t]{2}{*}{$0.000^{*}$} \\
\hline & $<50$ & 16,7 & 10,2 & 0,0 & 52,0 & \\
\hline \multirow[t]{2}{*}{ ÂNG. CÉRV. DIAFISÁRIO } & $\geq 50$ & 128,5 & 5,8 & 116,0 & 142,0 & \multirow[t]{2}{*}{$0.036^{*}$} \\
\hline & $<50$ & 130,2 & 5,8 & 116,0 & 145,0 & \\
\hline \multirow[t]{2}{*}{ OFFSET LATERAL } & $\geq 50$ & 37,6 & 3,3 & 29,0 & 44,0 & \multirow[t]{2}{*}{0.799} \\
\hline & $<50$ & 37,5 & 3,5 & 28,0 & 45,0 & \\
\hline \multirow[t]{2}{*}{ OFFSET VERTICAL } & $\geq 50$ & $-2,8$ & 8,1 & $-15,0$ & 24,0 & \multirow[t]{2}{*}{$0.028^{*}$} \\
\hline & $<50$ & 0,0 & 9,2 & $-16,0$ & 27,0 & \\
\hline \multirow[t]{2}{*}{ DIÂMETRO DO COLO } & $\geq 50$ & 25,2 & 3,7 & 18,0 & 37,0 & 0.947 \\
\hline & $<50$ & 25,2 & 4,2 & 17,0 & 37,0 & \\
\hline Corte axial & & & & & & \\
\hline ANTEVERSÃO ACETABULAR & $\geq 50$ & 22,6 & 6,5 & 7,0 & 44,0 & $0.003^{*}$ \\
\hline & $<50$ & 19,9 & 6,3 & 8,0 & 47,0 & \\
\hline AASA & $\geq 50$ & 65,4 & 10,5 & 35,0 & 87,0 & $0.002^{*}$ \\
\hline & $<50$ & 61,3 & 8,6 & 26,0 & 92,0 & \\
\hline PASA & $\geq 50$ & 110,4 & 14,8 & 80,0 & 159,0 & $0.000^{*}$ \\
\hline & $<50$ & 101,6 & 11,7 & 71,0 & 159,0 & \\
\hline HASA & $\geq 50$ & 176,0 & 20,9 & 132,0 & 234,0 & $0.000^{*}$ \\
\hline & $<50$ & 163,2 & 15,8 & 129,0 & 252,0 & \\
\hline DIÂMETRO CEFÁLICO & $\geq 50$ & 41,5 & 3,7 & 32,0 & 50,0 & 0.337 \\
\hline & $<50$ & 42,1 & 4,2 & 33,0 & 52,0 & \\
\hline DIÂMETRO ACETABULAR & $\geq 50$ & 52,3 & 4,2 & 41,0 & 60,0 & 0.616 \\
\hline & $<50$ & 52,6 & 3,9 & 42,0 & 61,0 & \\
\hline OFFSET CABEÇA COLO & $\geq 50$ & 9,2 & 3,0 & 4,0 & 28,0 & 0.403 \\
\hline & $<50$ & 9,5 & 2,5 & 5,0 & 21,0 & \\
\hline ÂNGULO ALFA ANTERIOR & $\geq 50$ & 48,2 & 7,6 & 30,0 & 71,0 & 0.526 \\
\hline & $<50$ & 47,6 & 6,7 & 30,0 & 67,0 & \\
\hline
\end{tabular}

Abreviaturas: AASA, ângulo do setor anterior do acetábulo; HASA, ângulo do setor horizontal do acetábulo; PASA, ângulo do setor posterior do acetábulo.

Fonte: Protocolo de pesquisa (2019). 
Estudo antropométrico tomográfico do quadril em uma população regional brasileira Busato et al. 237

Tabela 4 Medidas antropométricas em função do sexo

\begin{tabular}{|c|c|c|c|c|c|c|}
\hline VARIÁVEL & Sexo & Média & Desvio padrão & Mínimo & Máximo & Valor de $p$ \\
\hline \multicolumn{7}{|l|}{ CORTE CORONAL } \\
\hline \multirow[t]{2}{*}{ SHARP } & $\mathrm{F}$ & 40,5 & 5,4 & 28,0 & 63,0 & \multirow[t]{2}{*}{0.636} \\
\hline & $\mathrm{M}$ & 40,2 & 4,3 & 27,0 & 49,0 & \\
\hline \multirow[t]{2}{*}{ TONNIS } & $\mathrm{F}$ & 2,7 & 6,8 & $-13,0$ & 35,0 & \multirow[t]{2}{*}{0.223} \\
\hline & $M$ & 3,9 & 6,9 & $-11,0$ & 50,0 & \\
\hline \multirow[t]{2}{*}{ PROFUNDIDADE } & $\mathrm{F}$ & 18,3 & 2,7 & 12,0 & 25,0 & \multirow[t]{2}{*}{$0.004^{*}$} \\
\hline & $\mathrm{M}$ & 19,5 & 3,1 & 11,0 & 30,0 & \\
\hline \multirow[t]{2}{*}{ CE LATERAL } & $\mathrm{F}$ & 35,5 & 9,5 & 16,0 & 64,0 & \multirow[t]{2}{*}{$0.013^{*}$} \\
\hline & M & 32,6 & 7,1 & 15,0 & 54,0 & \\
\hline \multirow[t]{2}{*}{ CE MEDIAL } & $\mathrm{F}$ & 33,7 & 8,1 & 17,0 & 62,0 & \multirow[t]{2}{*}{0.650} \\
\hline & $\mathrm{M}$ & 33,2 & 8,9 & 13,0 & 66,0 & \\
\hline \multirow[t]{2}{*}{ ARCO ACETABULAR } & $\mathrm{F}$ & 68,7 & 12,2 & 47,0 & 103,0 & \multirow[t]{2}{*}{$0.046^{*}$} \\
\hline & $M$ & 65,5 & 10,2 & 38,0 & 103,0 & \\
\hline \multirow[t]{2}{*}{ ÂNG. DELTA: } & $\mathrm{F}$ & 28,4 & 9,2 & 5,0 & 52,0 & \multirow[t]{2}{*}{0.372} \\
\hline & M & 27,3 & 8,5 & 5,0 & 47,0 & \\
\hline \multirow[t]{2}{*}{ ÍND. EXTRUSÃO } & $\mathrm{F}$ & 11,4 & 9,6 & 0,0 & 52,0 & \multirow[t]{2}{*}{$0.001^{*}$} \\
\hline & $\mathrm{M}$ & 16,0 & 9,6 & 0,0 & 50,0 & \\
\hline \multirow[t]{2}{*}{ ÂNG. CÉRV. DIAFISÁRIO } & $\mathrm{F}$ & 129,0 & 6,3 & 116,0 & 142,0 & \multirow[t]{2}{*}{0.390} \\
\hline & $\mathrm{M}$ & 129,7 & 5,5 & 116,0 & 145,0 & \\
\hline \multirow[t]{2}{*}{ OFFSET LATERAL } & $\mathrm{F}$ & 36,5 & 3,6 & 28,0 & 45,0 & \multirow[t]{2}{*}{$0.000^{*}$} \\
\hline & M & 38,3 & 3,1 & 30,0 & 45,0 & \\
\hline \multirow[t]{2}{*}{ OFFSET VERTICAL } & $\mathrm{F}$ & $-2,1$ & 7,8 & $-15,0$ & 15,0 & \multirow[t]{2}{*}{0.265} \\
\hline & $\mathrm{M}$ & $-0,7$ & 9,4 & $-16,0$ & 27,0 & \\
\hline \multirow[t]{2}{*}{ DIÂMETRO DO COLO } & $\mathrm{F}$ & 23,7 & 3,6 & 17,0 & 32,0 & $0.000^{*}$ \\
\hline & $\mathrm{M}$ & 26,4 & 3,8 & 17,0 & 37,0 & \\
\hline CORTE AXIAL & & & & & & \\
\hline VERSÃO ACET. & $\mathrm{F}$ & 22,5 & 6,8 & 7,0 & 44,0 & $0.012^{*}$ \\
\hline & $\mathrm{M}$ & 20,2 & 6,1 & 8,0 & 47,0 & \\
\hline AASA & $\mathrm{F}$ & 63,6 & 9,6 & 35,0 & 86,0 & $0.742 \mathrm{~ns}$ \\
\hline & $\mathrm{M}$ & 63,1 & 9,8 & 26,0 & 92,0 & \\
\hline PASA & $\mathrm{F}$ & 107,8 & 13,6 & 73,0 & 159,0 & $0.086 \mathrm{~ns}$ \\
\hline & $\mathrm{M}$ & 104,4 & 14,1 & 71,0 & 159,0 & \\
\hline HASA & $\mathrm{F}$ & 171,8 & 19,0 & 129,0 & 234,0 & $0.125 \mathrm{~ns}$ \\
\hline & M & 167,5 & 19,7 & 129,0 & 252,0 & \\
\hline DIÂM. DA CABEÇA & $\mathrm{F}$ & 39,4 & 3,2 & 32,0 & 50,0 & $0.000^{*}$ \\
\hline & $\mathrm{M}$ & 43,6 & 3,5 & 36,0 & 52,0 & \\
\hline DIÂM. DO ACETÁBULO & $\mathrm{F}$ & 50,0 & 4,0 & 41,0 & 60,0 & $0.000^{*}$ \\
\hline & $\mathrm{M}$ & 54,2 & 3,0 & 45,0 & 61,0 & \\
\hline OFFSET CABEÇA-COLO & $\mathrm{F}$ & 8,9 & 2,3 & 4,0 & 14,0 & $0.055 \mathrm{~ns}$ \\
\hline & $M$ & 9,7 & 3,0 & 4,0 & 28,0 & \\
\hline ÂNG. ALFA ANTERIOR & $\mathrm{F}$ & 47,6 & 7,4 & 30,0 & 71,0 & $0.593 \mathrm{~ns}$ \\
\hline & M & 48,1 & 6,9 & 30,0 & 65,0 & \\
\hline
\end{tabular}

Abreviaturas: AASA, ângulo do setor anterior do acetábulo; HASA, ângulo do setor horizontal do acetábulo; PASA, ângulo do setor posterior do acetábulo.

FONTE: PROTOCOLO DE PESQUISA (2019). 
Tabela 5 Medidas antropométricas do canal femoral em função do sexo

\begin{tabular}{|c|c|c|c|c|c|c|}
\hline Variável & Sexo & Média & Desvio padrão & Mínimo & Máximo & Valor de $p$ \\
\hline \multirow[t]{2}{*}{$\mathrm{AP}:+2 \mathrm{CM}$} & $\mathrm{F}$ & 33,1 & 5,5 & 22,0 & 52,0 & \multirow[t]{2}{*}{$0.000^{*}$} \\
\hline & $M$ & 36,2 & 6,1 & 20,0 & 55,0 & \\
\hline \multirow[t]{2}{*}{$\mathrm{ML}:+2 \mathrm{CM}$} & $\mathrm{F}$ & 40,8 & 6,2 & 29,0 & 62,0 & \multirow[t]{2}{*}{0.747} \\
\hline & $M$ & 40,5 & 6,3 & 22,0 & 60,0 & \\
\hline \multirow[t]{2}{*}{ AP: superior } & $F$ & 29,3 & 4,8 & 20,0 & 45,0 & \multirow[t]{2}{*}{$0.000^{*}$} \\
\hline & $M$ & 32,3 & 4,4 & 22,0 & 44,0 & \\
\hline \multirow[t]{2}{*}{ ML: superior } & $\mathrm{F}$ & 35,6 & 4,2 & 24,0 & 50,0 & \multirow[t]{2}{*}{$0.001^{*}$} \\
\hline & $M$ & 37,7 & 4,4 & 25,0 & 49,0 & \\
\hline \multirow[t]{2}{*}{ AP: ápice } & $F$ & 26,4 & 4,8 & 17,0 & 40,0 & \multirow[t]{2}{*}{$0.014^{*}$} \\
\hline & $M$ & 28,1 & 5,0 & 18,0 & 40,0 & \\
\hline \multirow[t]{2}{*}{ ML: ápice } & $\mathrm{F}$ & 36,3 & 5,1 & 19,0 & 50,0 & \multirow[t]{2}{*}{$0.008^{*}$} \\
\hline & $M$ & 38,6 & 6,3 & 16,0 & 51,0 & \\
\hline \multirow[t]{2}{*}{ AP: inferior } & $\mathrm{F}$ & 22,2 & 4,2 & 14,0 & 32,0 & \multirow[t]{2}{*}{0.147} \\
\hline & $M$ & 23,0 & 3,8 & 12,0 & 35,0 & \\
\hline \multirow[t]{2}{*}{ ML: inferior } & $\mathrm{F}$ & 24,2 & 5,3 & 15,0 & 42,0 & \multirow[t]{2}{*}{0.658} \\
\hline & $M$ & 24,5 & 4,8 & 15,0 & 41,0 & \\
\hline \multirow[t]{2}{*}{ AP: - 2CM } & $\mathrm{F}$ & 17,6 & 3,3 & 11,0 & 28,0 & \multirow[t]{2}{*}{0.595} \\
\hline & $M$ & 17,9 & 3,3 & 10,0 & 28,0 & \\
\hline \multirow[t]{2}{*}{ ML: - 2CM } & $\mathrm{F}$ & 17,8 & 2,9 & 11,0 & 25,0 & \multirow[t]{2}{*}{0.461} \\
\hline & $M$ & 18,2 & 3,7 & 12,0 & 38,0 & \\
\hline
\end{tabular}

Abreviaturas: AP: Medida ânteroposterior; ML: Medida médio-lateral.

Fonte: Protocolo de pesquisa (2019).

Em outra pesquisa, ${ }^{1}$ os parâmetros médios observados foram os seguintes: ângulo de Sharp $39,2^{\circ}$ (menor do que o achado em nossa pesquisa, $40,3^{\circ}$ ), ângulo centro-borda lateral $32,7^{\circ}$ (menor do que o achado em nossa pesquisa, $33,8^{\circ}$ ), ângulo cérvico-diafisário $139,5^{\circ}$ (maior do que o achado em nossa pesquisa, $129,4^{\circ}$ ), versão acetabular $18,2^{\circ}$ (inferior ao achado em nossa pesquisa, 21,2), profundidade acetabular $25 \mathrm{~mm}$ (muito inferior ao encontrado em nossa pesquisa $19 \mathrm{~mm}$ ).

Nas medidas descritas por Anda et al. ${ }^{15}$, os arcos setoriais anterior e posterior do quadril, detectamos médias muito semelhantes àquelas descritas em seu estudo, com uma diferença de apenas $0,3^{\circ}$ no AASA e $0,9^{\circ}$ no PASA.

Sobre o diâmetro do acetábulo e da cabeça femoral, nossa população obteve médias menores, de 41,8 e 52,4 , respectivamente, contra $45,3 \mathrm{~mm}$ e $52,6 \mathrm{~mm}$, segundo estudo de Hauser et al. ${ }^{20}$

Sobre o offset lateral, medida de interesse direto para reconstrução biomecânica na artroplastia do quadril, Husmann et al demonstraram demonstrou média de $40,5 \mathrm{~mm}$, enquanto em nossa população, esta média foi de $37,6 \mathrm{~mm}$, variando de 28 a $45 \mathrm{~mm}$.

A análise conjunta de nossos resultados, especificamente no grupo acima de 50 anos, nos permite ponderar sobre o aprofundamento acetabular que acompanha o processo de envelhecimento. Este fato foi demonstrado neste grupo pelas medidas angulares significativamente maiores dos setores horizontais do acetábulo, do ângulo centro-borda e do arco acetabular, acompanhados de menor índice de extrusão. Podemos ainda notar uma varização, que foi demonstrada pela diminuição do ângulo cérvico-diafisário e do offset vertical, sendo que este último teve valor médio negativo nesta subamostra.

Quanto à forma do canal femoral, a análise dos dados obtidos com as medidas de diâmetro endosteal do canal femoral permitem esboçar um formato médio da região metafisária, o que seria de suma importância para um implante protético femoral personalizado para a amostra. Os valores médios obtidos são comparáveis àqueles obtidos por Noble et al.; ${ }^{17}$ porém, em nossa amostra, verificou-se que estas medidas são mais variáveis do que a maioria dos estudos prévios. Ainda, em relação a este estudo, também houve diferenças nas medidas do diâmetro do colo do fêmur. Noble et al. reportaram um diâmetro médio de $16,5 \mathrm{~mm}$, variando de 10 a $22 \mathrm{~mm}$, enquanto nós encontramos média de $25,2 \mathrm{~mm}$ e medidas que variaram entre 17 e $37 \mathrm{~mm}$. Especulamos que esta diferença possa ser decorrente da multitude étnica encontrada em nosso país. Tal variabilidade impacta diretamente no desenho de implantes céfalo-medulares com parafusos anti rotatórios. Seria desejável uma ampla gama de tamanhos disponíveis para atender à variedade anatômica de nossa população.

O estudo atual possui limitações inerentes ao seu desenho retrospectivo, além de não termos analisado se os casos 
Tabela 6 Medidas antropométricas do canal femoral em função da idade.

\begin{tabular}{|c|c|c|c|c|c|c|}
\hline Variável & Faixa Etária & Média & Desvio padrão & Mínimo & Máximo & Valor de $p$ \\
\hline \multirow[t]{2}{*}{$\mathrm{AP}:+2 \mathrm{CM}(\mathrm{mm})$} & $\geq 50$ & 35,0 & 5,5 & 22,0 & 52,0 & \multirow[t]{2}{*}{0.780} \\
\hline & $<50$ & 34,8 & 6,5 & 20,0 & 55,0 & \\
\hline \multirow[t]{2}{*}{$\mathrm{ML}:+2 \mathrm{CM}(\mathrm{mm})$} & $\geq 50$ & 40,2 & 6,9 & 22,0 & 62,0 & \multirow[t]{2}{*}{0.323} \\
\hline & $<50$ & 41,0 & 5,6 & 28,0 & 57,0 & \\
\hline \multirow[t]{2}{*}{ AP: superior $(\mathrm{mm})$} & $\geq 50$ & 30,7 & 4,6 & 22,0 & 42,0 & \multirow[t]{2}{*}{0.386} \\
\hline & $<50$ & 31,3 & 5,0 & 20,0 & 45,0 & \\
\hline \multirow[t]{2}{*}{$\mathrm{ML}$ : superior $(\mathrm{mm})$} & $\geq 50$ & 36,9 & 3,7 & 28,0 & 46,0 & \multirow[t]{2}{*}{0.880} \\
\hline & $<50$ & 36,8 & 5,0 & 24,0 & 50,0 & \\
\hline \multirow[t]{2}{*}{ AP: ápice (mm) } & $\geq 50$ & 27,0 & 4,7 & 18,0 & 38,0 & \multirow[t]{2}{*}{0.334} \\
\hline & $<50$ & 27,7 & 5,2 & 17,0 & 40,0 & \\
\hline \multirow[t]{2}{*}{ ML: ápice (mm) } & $\geq 50$ & 38,1 & 4,6 & 25,0 & 51,0 & \multirow[t]{2}{*}{0.249} \\
\hline & $<50$ & 37,1 & 6,9 & 16,0 & 50,0 & \\
\hline \multirow[t]{2}{*}{ AP: inferior (mm) } & $\geq 50$ & 22,5 & 3,8 & 12,0 & 35,0 & \multirow[t]{2}{*}{0.725} \\
\hline & $<50$ & 22,7 & 4,2 & 14,0 & 35,0 & \\
\hline \multirow[t]{2}{*}{$\mathrm{ML}$ : inferior (mm) } & $\geq 50$ & 24,5 & 4,5 & 18,0 & 40,0 & \multirow[t]{2}{*}{0.723} \\
\hline & $<50$ & 24,3 & 5,4 & 15,0 & 42,0 & \\
\hline \multirow[t]{2}{*}{ AP: $-2 \mathrm{CM}(\mathrm{mm})$} & $\geq 50$ & 17,5 & 2,9 & 12,0 & 27,0 & \multirow[t]{2}{*}{0.372} \\
\hline & $<50$ & 18,0 & 3,7 & 10,0 & 28,0 & \\
\hline \multirow[t]{2}{*}{$\mathrm{ML}:-2 \mathrm{CM}(\mathrm{mm})$} & $\geq 50$ & 18,3 & 3,2 & 12,0 & 29,0 & \multirow[t]{2}{*}{0.315} \\
\hline & $<50$ & 17,8 & 3,6 & 11,0 & 38,0 & \\
\hline
\end{tabular}

Abreviaturas: AP: Medida ânteroposterior; ML: Medida médio-lateral.

Fonte: Protocolo de pesquisa (2019).

apresentavam alguma sintomatologia ou qual foi o motivo da realização do exame de imagem. Ainda, as medidas por examinador único podem ser suscetíveis a variação, embora a TAC já tenha mostrado boa confiança intra e inter observador em algumas medidas. Como pontos positivos desta pesquisa ressaltamos que este estudo demonstrou de maneira inédita médias antropométricas detalhadas da articulação coxofemoral de uma população brasileira. Demonstramos também que ocorrem variações entre pacientes de faixas etárias diferentes. $\mathrm{O}$ presente protocolo de estudo poderia ser facilmente replicado em estudos multicêntricos, que julgamos necessários, para que se possa abranger uma amostra maior e mais diversificada de outras regiões do Brasil.

\section{Conclusões}

O presente estudo caracterizou detalhadamente a antropometria da articulação coxo-femoral de uma população regional brasileira. Demonstrou ainda diferenças morfológicas significativas do quadril entre diferentes faixas etárias e sexos.

\section{Suporte Financeiro}

Não houve suporte financeiro de fontes públicas, comerciais, ou sem fins lucrativos.

Conflito de Interesses

Os autores declaram não haver conflito de interesses.

\section{Referências}

1 Saikia KC, Bhuyan SK, Rongphar R. Anthropometric study of the hip joint in northeastern region population with computed tomography scan. Indian J Orthop 2008;42(03):260-266

2 Sengodan VC, Sinmayanantham E, Kumar JS. Anthropometric analysis of the hip joint in South Indian population using computed tomography. Indian J Orthop 2017;51(02):155-161

3 Abbas K, Murtaza G, Umer M, Rashid H, Qadir I. Complications of total hip replacement. J Coll Physicians Surg Pak 2012;22(09):575-578

4 Banerjee S, Faizan A, Nevelos J, et al. Innovations in hip arthroplasty three-dimensional modeling and analytical technology (SOMA). Surg Technol Int 2014;24:288-294

5 Parratte S, Flecher X, Vesin O, Brunet C, Aubaniac J, Argenson J. Anatomy of the hip before total hip arthroplasty: A tridimentionnal Ct study of 469 patients. Orthop Proc 2018; 92-B Supp II. Disponível em: https://online.boneandjoint.org.uk/doi/abs/ 10.1302/0301-620X.92BSUPP_II.0920274d

6 Kang AC, Gooding AJ, Coates MH, Goh TD, Armour P, Rietveld J. Computed tomography assessment of hip joints in asymptomatic individuals in relation to femoroacetabular impingement. Am J Sports Med 2010;38(06):1160-1165

7 Abel MF, Sutherland DH, Wenger DR, Mubarak SJ. Evaluation of CT scans and 3-D reformatted images for quantitative assessment of the hip. J Pediatr Orthop 1994;14(01):48-53

8 Murtha PE, Hafez MA, Jaramaz B, DiGioia AM III. Variations in acetabular anatomy with reference to total hip replacement. J Bone Joint Surg Br 2008;90(03):308-313

9 Stem ES, O'Connor MI, Kransdorf MJ, Crook J. Computed tomography analysis of acetabular anteversion and abduction. Skeletal Radiol 2006;35(06):385-389 
10 Mineta K, Goto T, Wada K, et al. CT-based morphological assessment of the hip joint in Japanese patients: association with radiographic predictors of femoroacetabular impingement. Bone Joint J 2016;98-B(09):1167-1174

11 Husmann O, Rubin PJ, Leyvraz PF, de Roguin B, Argenson JN. Three-dimensional morphology of the proximal femur. J Arthroplasty 1997;12(04):444-450

12 Pasquier G, Ducharne G, Ali ES, Giraud F, Mouttet A, Durante E. Total hip arthroplasty offset measurement: is C T scan the most accurate option? Orthop Traumatol Surg Res 2010;96(04): 367-375

13 Dandachli W, Ul Islam S, Tippett R, Hall-Craggs MA, Witt JD. Analysis of acetabular version in the native hip: comparison between 2D axial CT and 3D CT measurements. Skeletal Radiol 2011;40(07):877-883

14 Lepage-Saucier M, Thiéry C, Larbi A, Lecouvet FE, Vande Berg BC, Omoumi P. Femoroacetabular impingement: normal values of the quantitative morphometric parameters in asymptomatic hips. Eur Radiol 2014;24(07):1707-1714
15 Anda S, Terjesen T, Kvistad KA. Computed tomography measurements of the acetabulum in adult dysplastic hips: which level is appropriate? Skeletal Radiol 1991;20(04):267-271

16 Tannast M, Hanke MS, Zheng G, Steppacher SD, Siebenrock KA. What are the radiographic reference values for acetabular underand overcoverage? Clin Orthop Relat Res 2015;473(04):1234-1246

17 Noble PC, Alexander JW, Lindahl LJ, Yew DT, Granberry WM, Tullos HS. The anatomic basis of femoral component design. Clin Orthop Relat Res 1988;(235):148-165

18 Beltran LS, Mayo JD, Rosenberg ZS, et al. Fovea alta on MR images: is it a marker of hip dysplasia in young adults? AJR Am J Roentgenol 2012;199(04):879-883

19 Tannast M, Fritsch S, Zheng G, Siebenrock KA, Steppacher SD. Which radiographic hip parameters do not have to be corrected for pelvic rotation and tilt? Clin Orthop Relat Res 2015;473(04):1255-1266

20 Hauser DL, Fox JC, Sukin D, Mudge B, Coutts RD. Anatomic variation of structural properties of periacetabular bone as a function of age. A quantitative computed tomography study. J Arthroplasty 1997;12(07):804-811 